\title{
Sources of Apical Defects on a High-Sensitivity Cardiac Camera: Experiences from a Practice Performance Assessment
}

\author{
Leo L. Tsai ${ }^{1}$, Kevin J. Donohoe ${ }^{1}$, Margaret K. Stokes ${ }^{1}$, Thomas H. Hauser ${ }^{2}$, Gerald M. Kolodny ${ }^{1}$, Thomas C. Hill ${ }^{1}$, \\ and J. Anthony Parker ${ }^{1}$ \\ ${ }^{1}$ Department of Radiology, Beth Israel Deaconess Medical Center, Boston, Massachusetts; and ${ }^{2}$ Cardiovascular Institute, Beth Israel \\ Deaconess Medical Center, Boston, Massachusetts
}

\begin{abstract}
Apical perfusion artifacts seen on a high-sensitivity camera warranted a practice performance assessment to evaluate contributions from soft-tissue attenuation, patient positioning, and image processing techniques. Methods: Cardiac perfusion studies $(n=534)$ spanning 5 mo were retrospectively reviewed. Images were acquired with the patient in the upright position, and attenuation correction was used. Regression analysis and contingency tables correlated clinical data to the presence of apical artifacts. Results: There was a positive correlation of with female sex $\left(\chi^{2}=32, P<0.001\right)$, degree of overlying soft tissues $\left(\chi^{2}=20, P<0.002\right)$, and breast cleavage $\left(\chi^{2}=7, P<\right.$ 0.008 ) and a negative correlation with angiography-confirmed disease $\left(\chi^{2}=6, P<0.02\right)$. There was moderate interobserver agreement between 2 observers in determining the presence of apical defects $(\kappa=0.44,95 \%$ confidence interval $=0.19-0.69$ ), and there was a perceived improvement of apical defects using fewer iterative updates $\left(\chi^{2}=8, P<0.003\right)$. Conclusion: An understanding of sources contributing to imaging artifacts is a crucial portion of quality assessment in radiology and nuclear medicine. A practice performance assessment study at our institution showed that apical artifacts on a new-generation cardiac camera can be partially attributed to overlying soft-tissue attenuation and ameliorated by altering the reconstruction.
\end{abstract}

Key Words: cardiology (basic/technical); instrumentation; quality assurance; iterative reconstruction; myocardial perfusion; practice performance assessment

J Nucl Med Technol 2013; 41:197-202

DOI: 10.2967/jnmt.113.124198

$\mathbf{M}$ yocardial perfusion imaging is susceptible to a wide variety of artifacts that mimic perfusion defects (1-3). These can arise from several sources, including the patient, imaging system, and processing techniques. Characterization of these defects is important, because artifacts can lower the specificity for detection of true cardiac perfusion abnormalities.

\footnotetext{
Received Apr. 15, 2013; revision accepted Jun. 28, 2013.

For correspondence or reprints contact: Leo L. Tsai, Department of Radiology, Beth Israel Deaconess Medical Center, 330 Brookline Ave., West Campus, 3rd Floor, Boston, MA 02215.

E-mail: Itsai1@bidmc.harvard.edu

Published online Aug. 15, 2013.

COPYRIGHT @ 2013 by the Society of Nuclear Medicine and Molecular Imaging, Inc.
}

Examinations performed on a single high-sensitivity, dedicated cardiac camera in our institution using resolution recovery appeared to result in a substantially higher rate of apparent apical perfusion defects (\#17 of the American Heart Association segment model) (4). These artifacts were only partially, and usually marginally, ameliorated by attenuation correction. The objective of this practice performance assessment project (http://en.wikipedia.org/wiki/ American_Board_of_Medical_Specialties) was to measure the rate and severity of these defects and to characterize the clinical correlates on this single system. By considering the effects of reconstruction algorithms, we developed a plan to ameliorate these defects. We then remeasured defect severity after modifying the reconstruction parameters.

\section{MATERIALS AND METHODS}

A quality initiative waiver was approved by the institutional review board for this retrospective study.

\section{Practice Performance Assessment}

A goal of maintenance of certification part 4 projects is the identification and measurement of possible problems in a practice, followed by implementation and remeasurement of improvements. The subjective consensus in our department was that there was a problem with apical defects using a new high-sensitivity camera. After selection of a potential problem, we performed an initial measurement to evaluate the problem. We then identified and implemented an intervention. Finally, we remeasured to evaluate the intervention. This report describes our practice performance assessment project.

\section{Patient Population}

The sample size was chosen by conservatively estimating that $5 \%$ of individuals undergoing a cardiac perfusion study at our institution had a true focal perfusion defect. We calculated the sample size necessary to achieve a standard power of 0.8 for a detectable change of $2.5 \%$ from any correlate (single tail with $\alpha=$ $0.05)$. This calculation resulted in a sample size requirement of 500 . We satisfied this requirement by including all myocardial perfusion studies performed at our institution from August to December 2009, which totaled 534 patients (309 men and 225 women).

\section{Image Acquisition}

Imaging was performed on a high-sensitivity, dedicated cardiac SPECT/CT camera (Cardius XACT; Digirad) at our home institution. Rest imaging was performed with $370 \mathrm{MBq}(10 \mathrm{mCi})$ of ${ }^{99 \mathrm{~m} T c-s e s t a m i b i}$ with gating. A tomographic image of the chest 
was acquired for attenuation correction. Exercise stress was performed on a treadmill; pharmacologic stress imaging was performed with persantine or dobutamine. ${ }^{99 \mathrm{~m}} \mathrm{Tc}$-sestamibi $(1,110$ $\mathrm{MBq}[30 \mathrm{mCi}]$ ) was injected with stress. Imaging was performed with the patient upright, sitting on a chair, with both arms placed on an armrest at shoulder height. A built-in strap was used to secure the patient to the imaging chair. The system uses three $20 \times 15 \mathrm{~cm}$ detector heads with high-sensitivity fan beam collimators. These function as a triple-head system for both emission and transmission scans. The latter configuration is used in conjunction with a collimated fluorescence $\mathrm{x}$-ray emitted from a lead target fluxed by a narrow beam from an x-ray tube, allowing for rapid transmission scans for attenuation correction (5). This configuration is unique in that the patient chair rotates; the patient chair is positioned so that the heart is centered at the axis of rotation. Images were acquired at $30 \mathrm{~s}$ per frame at rest and $25 \mathrm{~s}$ per frame at stress.

Motion correction was used when deemed appropriate after the raw images were first reviewed. An attenuation map was obtained for every examination, and attenuation correction was used for every scan. As with our standard diagnostic protocol, both attenuation-corrected and non-attenuation-corrected images were provided to the readers.

\section{Apical Defect and Soft-Tissue Scoring}

The presence and severity of apical defects was scored for all studies by 1 nuclear medicine physician who was masked to all patient data except for the images. Normal examinations were given a severity score of 1 . The appearance of the defects was judged as true or artifactual, and the degree of severity was ranked from 2 (mild) to 5 (severe). Apical artifacts were defined as decreased counts within segment 17 of the American Heart Association standard segmentation that were not attributable to true perfusion abnormalities. To limit the degree of selection bias, all cases of true perfusion defects as confirmed by angiography were included in the study, regardless of location or apical involvement. The amount of overlying soft tissues was scored on a scale of 0 for minimal or none, $\mathrm{A}$ for a small amount, and $\mathrm{E}$ for a very large amount. The presence of breast cleavage was scored as present or not present. Cleavage is defined as any perceptible separation between the breasts on the attenuation map, which provides axial CT reconstructions of the patient's chest.

\section{Additional Patient Data}

The following data were obtained from medical records if available: age, sex, peak heart rate and systolic blood pressure during the examination, any confirmed vessel occlusion by angiography, history of coronary artery bypass graft (CABG), smoking history, history of diabetes, body mass index, and total and highdensity lipoprotein cholesterol levels within the past year. Whenever possible, in patients who did not have coronary artery disease (CAD), the 10-y CAD risk was calculated using the Framingham study guidelines (6). Patients who were determined to have a greater than $10 \%$ risk under this calculation were labeled high risk.

\section{Analysis}

All statistics were performed using the JMP software package from SAS. A subset of 110 examinations that were interpreted as having a normal cardiac perfusion study was randomly selected for comparison to examinations demonstrating apical defects. Logistic regression analysis using a Pearson test was used to correlate the presence of apical defects with age and peak heart rate. Contingency tables using the Fisher exact test and forward stepwise regression analysis using Akaike information criterion were applied to correlate the patient sex, presence of CAD, amount of overlying soft tissues, presence of coronary vessel disease, and calculated high risk for CAD to the presence of perfusion defects or artifacts.

\section{Image Reconstruction}

Three-dimensional ordered-subset estimation maximization image reconstruction was performed on a workstation using proprietary software. The algorithm uses a slab-by-slab approach (nSPEED): each slab, consisting of several slices, is used in ordered-subset estimation maximization reconstruction, reducing overall processing time (7). Studies were performed with the recommended number of updates ( 2 iterations, 15 subsets). Our data were shared with the vendor, and 2 potential software solutions were suggested-reduction in the number of updates or removal of resolution recovery from the reconstruction algorithm. Both solutions were applied to a small group of patients, and the investigators selected the reduced-updates solution for more detailed evaluation. During the reevaluation of images with fewer iterative updates, 2 iterations with 10 subsets were used.

\section{Comparison of Different Reconstruction Methods}

A subset of 50 cardiac perfusion studies from the original patient set was selected. Twenty-five studies were interpreted as normal and served as controls, and 25 were interpreted as having artifactual apical defects by the primary observer. These studies were then randomized. The raw data for each study were processed using the same 3-dimensional ordered-subset estimation maximization method as in the original study but with the decreased updates. Original data and reprocessed image sets were presented together, but in randomized order, for interpretation by a second nuclear medicine physician, who was masked to results, clinical history, and patient identifiers. The interpreter was instructed to rate the severity of defect with a scale ranging from 1 (no defect) to 6 (severe) while spreading the scores over the full scale during evaluation. $\kappa$-values were calculated to assess for interobserver agreement between the primary and secondary interpreters in detecting the presence of an apical defect, with the goal of confirming interobserver agreement on the definition of the apical artifact. A contingency table using the Fisher exact test was applied to determine the significance of perceived improvements from reconstructions performed with fewer iterative updates.

\section{RESULTS}

A summary of the patient population is provided in Table 1. Ninety-six individuals (18.0\%) demonstrated apical defects. Of these, $66(68.8 \%)$ were scored as artifacts and $30(31.2 \%)$ as true perfusion defects. Figure 1 shows examples of apical defects and overlying soft tissues. There was no significant relationship between artifact occurrence and age $\left(\chi^{2}=0.01\right.$, $P<0.49)$ or peak heart rate $\left(\chi^{2}=0.07, P<0.79\right)$.

Using Pearson contingency analysis, we found a significant positive correlation with female sex $\left(\chi^{2}=32, P<\right.$ $0.001)$, the presence of overlying anterior chest soft tissues $\left(\chi^{2}=20, P<0.002\right)$, and the presence of breast cleavage $\left(\chi^{2}=7, P<0.008\right)$. Negative associations were seen between the artifacts and angiography-confirmed 1- to 3-vessel disease $\left(\chi^{2}=6, P<0.02\right)$ and elevated cardiac risk $(>10 \%$ 
TABLE 1

Summary of Patient Population and Results of Cardiac Perfusion Studies

\begin{tabular}{lccc}
\hline Total no. of... & $n$ & $\begin{array}{c}\text { Angiography-proven CAD } \\
\text { before examination }\end{array}$ & $\begin{array}{c}\text { Elevated risk of acute coronary } \\
\text { syndrome }(>10 \% \text { at } 10 \text { y) }\end{array}$ \\
\hline Patients & 534 & 48 & 58 \\
Men & 309 & 31 & 14 \\
Women & 225 & 17 & 44 \\
Apical defects & 96 & 25 & 35 \\
True apical defects & 30 & 14 & 0 \\
Artifactual apical defects & 66 & 11 & 35 \\
\hline
\end{tabular}

risk for acute coronary syndrome at $10 \mathrm{y}, \chi^{2}=15, P<$ 0.0001 ). These results are summarized in Table 2. A mosaic plot demonstrating the amount of overlying soft tissues against the presence of apical defects is shown in Figure 2.

Because of expected confounding bias among sex, softtissue attenuation, and cleavage, forward stepwise regression with Akaike information criterion was performed with all variables, then twice more without 1 or 2 of the variables. Regression analysis with all variables resulted in a best model with sex $\left(P<5 \times 10^{-7}\right)$ and elevated cardiac risk $(P<0.003)$. When sex was removed, the model converged on soft tissue $\left(P<3 \times 10^{-5}\right)$ and elevated cardiac risk $\left(<5 \times 10^{-4}\right)$. When both sex and soft tissue were removed, the model converged on cleavage $(P<0.002)$ and elevated cardiac risk $\left(<2 \times 10^{-5}\right)$.

Medical records for all cases with any perfusion defects (artifact or real) were reviewed up to 18 mo after the study, to look for additional diagnostic workup such as angiography or serum laboratory tests. In all studies that were interpreted as having only an apical artifact, no diagnosis of significant coronary vessel disease or acute coronary syndrome was made within the 18-mo window.

On the basis of the results of the measurement phase, a plan was formulated to alter the reconstruction process. Scans were reprocessed and then evaluated under the remeasurement phase of the practice performance assessment.

In the comparison of processing methods, there was moderate interobserver agreement when determining the presence of apical defects of the original images $(\kappa=0.44$,
$95 \%$ confidence interval $=0.19-0.69)$, with the second interpreter detecting an apical defect in 11 of the 25 control studies and in all within the artifact group (Tables 3 and 4). Within the control group, the second interpreter saw no change in 18 studies (72\%) and an improved apical defect in 6 cases $(24 \%)$. Within the artifact group, 8 studies were interpreted as unchanged $(32 \%)$ and 17 cases as improved $(68 \%)$. There was a significant perceived improvement of apical defects after reconstruction with fewer updates $\left(\chi^{2}=8, P<0.003\right)$. No study was interpreted as having a more severe defect with the modified reconstruction. A representative comparison of the 2 reconstruction methods, showing improvement of an apical defect, is demonstrated in Figure 3.

\section{DISCUSSION}

Because of the perception that some apical defects seen on myocardial perfusion imaging were artifactual on one of our cameras, a practice performance assessment project was undertaken. During the measurement phase, scans were interpreted for severity by a masked observer. During the assessment phase, defect severity was shown to correlate positively with factors related to attenuation and negatively with factors related to CAD. These findings supported the clinical perception that some of the defects were artifactual. A plan was formulated to alter reconstruction parameters to ameliorate these defects. The remeasurement phase of the project showed that the altered reconstruction parameters resulted in a reduction in perceived artifactual apical defects.

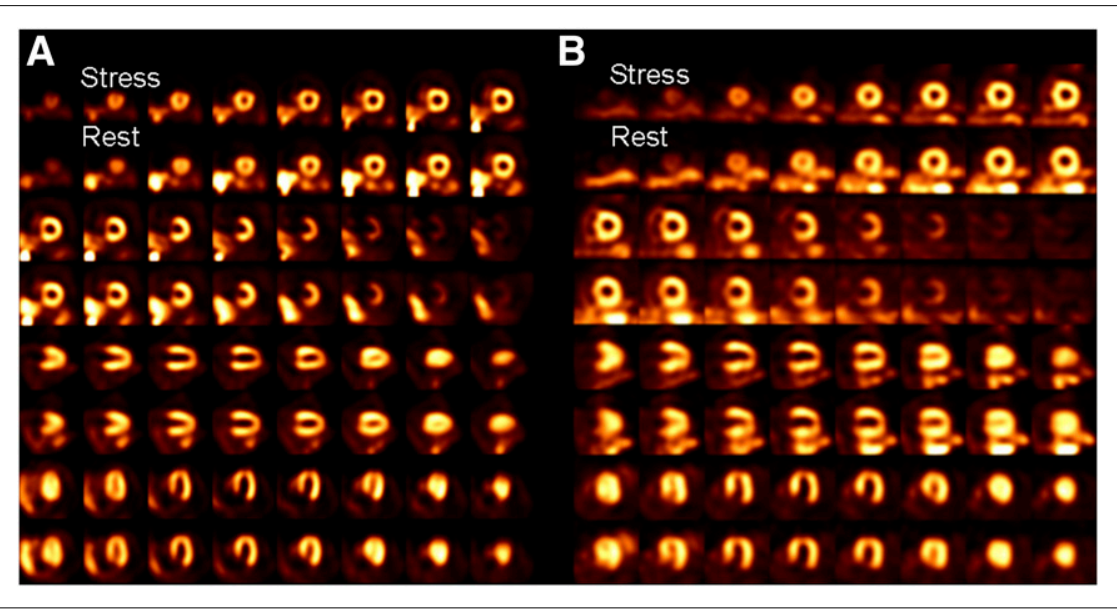

FIGURE 1. Example cardiac perfusion scans from 2 patients. (A) Scan from an 86-y-old woman with reversible apical defect. Coronary angiogram revealed a $90 \%$ stenosis of left anterior descending artery. (B) Scan from a 50-y-old woman demonstrating fixed apical defect. Gated images demonstrated normal wall motion and thickening. Subsequent clinical workup was negative for CAD. 
TABLE 2

Summary of Results Comparing Presence of Apical Artifacts with Clinical Correlates

\begin{tabular}{lcc}
\hline $\begin{array}{c}\text { Clinical correlates against presence of } \\
\text { apical artifacts }\end{array}$ & $\begin{array}{c}\text { Association and Pearson } \\
\chi^{2} \text { value }\end{array}$ & $\begin{array}{c}P \text { value, } \\
\text { Fisher exact test }\end{array}$ \\
\hline Female sex & Positive, 32.7 & $P<0.001$ \\
Degree of overlying soft-tissues & Positive, 19.5 & $P<0.002$ \\
Presence of breast cleavage & Positive, 7.15 & $P<0.008$ \\
Angiography-confirmed CAD & Negative, 6.88 & $P<0.02$ \\
Elevated cardiac risk $(>10 \%$ at $10 y)$ & Negative, 14.6 & $P<0.0001$ \\
\hline
\end{tabular}

Artifacts on myocardial perfusion imaging can arise from several sources, including the equipment used, processing techniques, positioning, and patient anatomy. In this study, we sought to understand the source of perceived increased apical artifacts using a high-sensitivity dedicated cardiac camera and its potential impact on patient outcome.

Attenuation is known to be a major cause of perfusion artifacts, because the heart is surrounded by many different structures with variable densities $(8-10)$. Our results show that soft-tissue attenuation was a contributor to the apical artifact, with breast tissue being a primary source. This conclusion is reflected in the strong association with the female sex in the logistical analysis. This finding is consistent with prior studies attributing breast attenuation to artifacts occurring within the left anterior descending artery, left circumflex territories, and apex $(3,11)$. Breast cleavage was also found to be a contributor in our study. Although a major confounder includes the positive relationship among female sex, presence of cleavage, and overall amount of breast tissue, stepwise multivariate regression did confirm independently significant relationships among these variables with the apical artifacts.

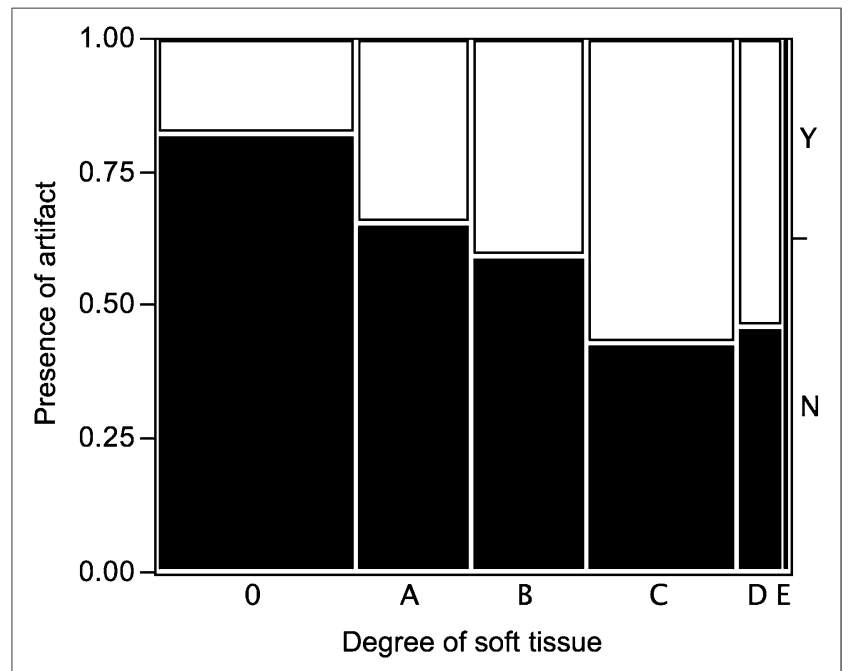

FIGURE 2. Mosaic plot demonstrating presence of apical artifacts against amount of soft tissues overlying chest wall. Both axes are scaled by fraction of individuals, compared with total number within each group. Amount of tissue ranges from minimal or none $(0)$, to very little $(A)$, to a very large amount $(E)$.
The camera that was evaluated obtains images with the patient in an upright sitting posture; most other cameras acquire images with the patient in decubitus or semierect positions. A recent study demonstrated substantially increased contribution to inferior and lateral wall attenuation within women who were imaged upright (12). This study does not focus on the apical artifacts that are seen on our system. Nevertheless, the study does suggest that there is a role in patient positioning and the artifacts detected on our system and also illustrates the contribution of breast tissue and correlation with female sex as a major factor. Indeed, in our experience with a small number of patients who have been imaged on both cameras in our department, we find there to be substantial differences in attenuation, but we do not have enough subjects to make any statistically meaningful conclusions. Further evaluation is hindered by the inability to perform imaging in both orientations on the same camera, limiting our ability to separate this effect from other camera-specific systematic errors.

The negative association between apical artifacts with confirmed CAD or increased risk of CAD likely reflects sampling bias. The ability to accurately differentiate true and artifactual perfusion defects is of clinical importance, because these artifacts may decrease the specificity of the examination. Cardiac motion is one of the most critical components used to differentiate artifacts from true defects; an apparent fixed-perfusion defect in a wall demonstrating normal kinetics and normal contractive thickening is generally attributed to soft-tissue attenuation. Indeed, of the studies that were clinically interpreted as having only soft-tissue attenuation, no subsequent diagnosis of significant coronary vessel disease was made within $18 \mathrm{mo}$ of the original examination.

Attenuation correction only ameliorated apical artifacts partially at best, and often only marginally, leading us to examine other contributors to their presence. In particular, we discovered that there was partial improvement of these artifacts when the number of iterative updates was reduced

TABLE 3

Interobserver Comparison for Detection of Apical Artifacts

\begin{tabular}{lcc}
\hline & \multicolumn{3}{c}{ Observer 1 } \\
\cline { 2 - 3 } Observer 2 & Normal & Artifact \\
\hline Normal & 11 & 0 \\
Artifact & 14 & 25 \\
\hline
\end{tabular}


TABLE 4

Comparison of Reconstruction Methods by Observer 2

\begin{tabular}{cccc}
\hline & Improved & Unchanged & Worse \\
\hline Normal & 7 & 18 & 0 \\
Artifact & 17 & 9 & 0 \\
\hline
\end{tabular}

during reconstruction. Iterative reconstruction methods have several advantages over traditional backprojection techniques; in particular, they allow more accurate attenuation, scattering, and depth-dependent resolution to be included (13-16). They also allow for more flexible incorporation of resolution recovery (17). However, iterative reconstruction leads to more complex noise amplification (18-21). The comparison between the standard reconstructions and those with fewer updates on our cardiac camera showed that there was an overall significant net decrease in apical artifacts. Furthermore, the second reviewer did not show any worsening of the artifact with decreased iterative updates. The instruction to distribute scores over the entire range may explain why the second observer scored apical defects more severely than the first observer. These data suggest that the reconstruction methods are indeed a contributor to the observed apical artifacts. Other factors may also contribute, because apical defects perceived to be artifactual still occurred with the modified reconstructions.

Any change in reconstruction methods could affect both real and false perfusion defects. This raises the concern that perhaps true perfusion defects may be masked by the updated reconstruction, and indeed one limitation of this study was the lack of quantification of such change. However, a formal study of diagnostic accuracy was beyond the scope of our practice performance assessment project. Diagnostic accuracy would be subject to many additional factors. Because true defects are generally larger than the apical defects studied, they might be less affected by a change in reconstruction. Patient history, gated data, and comparison between non-attenuation-corrected and attenuation-corrected images are examples of additional factors that might affect accuracy. We noted a perceived problem with our practice, and our practice performance assessment project has ameliorated that problem. During routine clinical follow-up with angiographic results, there is no perceived difference in our ability to detect true perfusion defects, but this possibility remains a limitation of this project.

This study did not focus on other contributors to artifacts. For example, diaphragmatic attenuation is a known source of artifacts, but the inferior wall is more likely affected than the apex (2). Subdiaphragmatic activity, mainly from the liver, also involves the inferior wall $(2,3,9,22)$ and is more susceptible in systems using filtered backprojection reconstructions rather than the iterative reconstructions (23). Lastly, this study was limited to 1 cardiac camera in a single institution, so systematic errors were potentially undetected, and potential solutions such as reduction of iterative updates may not be applicable to other departments or other systems. Our approach to a perceived practice problem provides an example to others of how to implement practice performance assessment projects in their own practice.

\section{CONCLUSION}

An apparent increase in number of apical defects on cardiac perfusion studies performed on a new-generation, high-sensitivity, dedicated cardiac camera was evaluated with a practice performance assessment project. Defects were associated with the amount of overlying soft tissues and negatively associated with markers of CAD. The severity of the defects was also influenced by the number of updates used in the iterative reconstruction. Reducing the number of iterative updates was shown to ameliorate the number of apical defects.

\section{DISCLOSURE}

No potential conflict of interest relevant to this article was reported.

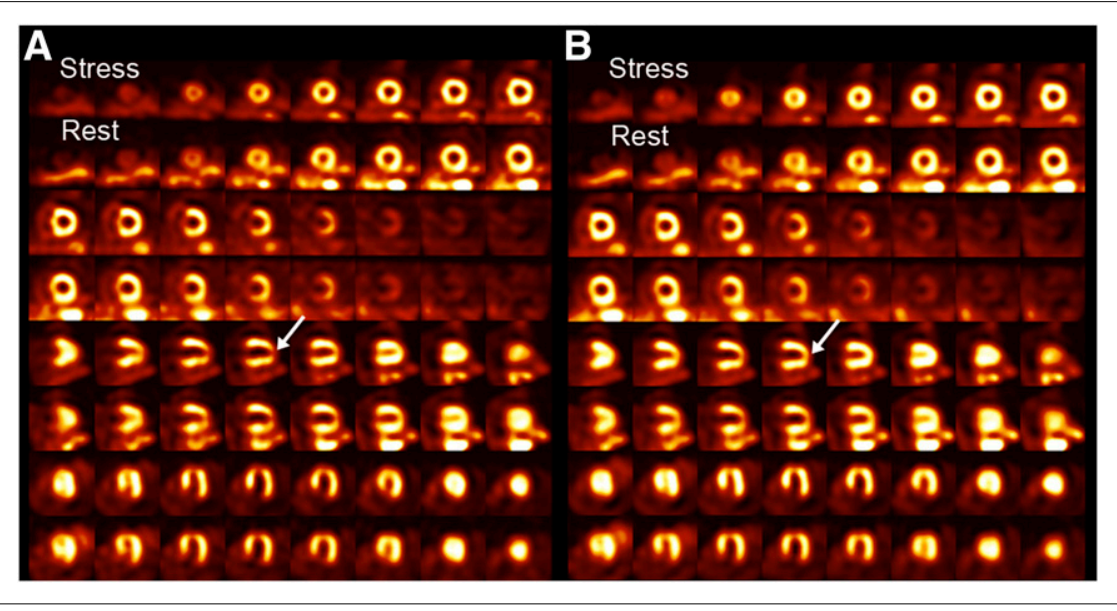

FIGURE 3. Cardiac perfusion examination demonstrating artifactual apical defects, with standard reconstruction $(A)$ and one with decreased iterative reconstruction (B). Both stress and rest images were acquired, with stress images located along upper rows. These images are attenuationcorrected. Arrows highlight area of apical defect improvement after adjusted reconstruction. 


\section{REFERENCES}

1. Burrell S, MacDonald A. Artifacts and pitfalls in myocardial perfusion imaging. J Nucl Med Technol. 2006;34:193-211.

2. Dvorak RA, Brown RK, Corbett JR. Interpretation of SPECT/CT myocardial perfusion images: common artifacts and quality control techniques. Radiographics. 2011;31:2041-2057.

3. Wackers FJ. Artifacts in planar and SPECT myocardial perfusion imaging. Am J Card Imaging. 1992;6:42-57, discussion 58.

4. Cerqueira MD, Weissman NJ, Dilsizian V, et al. Standardized myocardial segmentation and nomenclature for tomographic imaging of the heart: a statement for healthcare professionals from the Cardiac Imaging Committee of the Council on Clinical Cardiology of the American Heart Association. Circulation. 2002;105:539-542.

5. Bai C, Babla H, Kindem J, Conwell R, Weatherhead R. Evaluation of a fullyintegrated cardiac SPECT/VCT system using a common set of solid-state detectors for both emission and transm scans and a novel low dose lead fluorescence x-ray transm line source. 2007. http://www.digirad.com/downloads_2007/XACT\%20White \%20Paper\%20v7.pdf. Accessed July 23, 2013.

6. Wilson PW, D'Agostino RB, Levy D, Belanger AM, Silbershatz H, Kannel WB. Prediction of coronary heart disease using risk factor categories. Circulation. 1998;97:1837-1847.

7. Bai C, Conwell $\mathrm{R}$, Babla $\mathrm{H}$, et al. Improving image quality and imaging efficiency using nSPEEDSM three-dimensional image reconstruction in cardiac SPECT. 2007. http:// www.digirad.com/downloads_2007/nspeed_white_paper.pdf. Accessed July 23, 2013.

8. Hansen CL, Woodhouse S, Kramer M. Effect of patient obesity on the accuracy of thallium-201 myocardial perfusion imaging. Am J Cardiol. 2000;85:749-752.

9. Nuyts J, Dupont P, Van den Maegdenbergh V, Vleugels S, Suetens P, Mortelmans L. A study of the liver-heart artifact in emission tomography. J Nucl Med. 1995;36: $133-139$.

10. Germano G, Chua T, Kiat H, Areeda JS, Berman DS. A quantitative phantom analysis of artifacts due to hepatic activity in technetium- $99 \mathrm{~m}$ myocardial perfusion SPECT studies. J Nucl Med. 1994;35:356-359.
11. Manglos SH, Thomas FD, Gagne GM, Hellwig BJ. Phantom study of breast tissue attenuation in myocardial imaging. J Nucl Med. 1993;34:992-996.

12. Chawla D, Rahaby M, Amin AP, et al. Soft tissue attenuation patterns in stress myocardial perfusion SPECT images: a comparison between supine and upright acquisition systems. J Nucl Cardiol. 2011;18:281-290.

13. Bouwens L, Van de Walle R, Nuyts J, et al. Image-correction techniques in SPECT. Comput Med Imaging Graph. 2001;25:117-126.

14. Bruyant PP. Analytic and iterative reconstruction algorithms in SPECT. J Nucl Med. 2002;43:1343-1358.

15. Vandenberghe S, D'Asseler Y, Van de Walle R, et al. Iterative reconstruction algorithms in nuclear medicine. Comput Med Imaging Graph. 2001;25:105-111.

16. Hutton BF. Recent advances in iterative reconstruction for clinical SPECT/PET and CT. Acta Oncol. 2011;50:851-858.

17. Kappadath SC. Effects of voxel size and iterative reconstruction parameters on the spatial resolution of ${ }^{99 \mathrm{~m}}$ Tc SPECT/CT. J Appl Clin Med Phys. 2011;12:210-220.

18. Brambilla M, Cannillo B, Dominietto M, Leva L, Secco C, Inglese E. Characterization of ordered-subsets expectation maximization with $3 \mathrm{D}$ post-reconstruction Gauss filtering and comparison with filtered backprojection in ${ }^{99 \mathrm{~m} T c}$ SPECT. Ann Nucl Med. 2005;19:75-82.

19. Bai B, Kulon M, Esser P. Variations in SUV values with reconstruction algorithms [abstract]. J Nucl Med. 2011;52(suppl 1):2100.

20. Wang CX, Snyder WE, Bilbro G, Santago P. Performance evaluation of filtered backprojection reconstruction and iterative reconstruction methods for PET images. Comput Biol Med. 1998;28:13-24.

21. de Jonge FA, Blokland KA. Statistical tomographic reconstruction: how many more iterations to go? Eur J Nucl Med. 1999;26:1247-1250.

22. Pitman AG, Kalff V, Van Every B, Risa B, Barnden LR, Kelly MJ. Contributions of subdiaphragmatic activity, attenuation, and diaphragmatic motion to inferior wall artifact in attenuation-corrected Tc-99m myocardial perfusion SPECT. J Nucl Cardiol. 2005;12:401-409.

23. Hsu B, Kao CH, Hung GU. Recognition of image artifacts on myocardial perfusion SPECT enhanced by iterative reconstruction. Clin Nucl Med. 2010;35:445-447. 